\title{
The Goddard Fabry-Perot Imager
}

\author{
Richard Gelderman \\ National Research Council Research Associate, \\ NASA/GSFC Lab for Astronomy \& Solar Physics, Greenbelt, \\ $M D 20771$
}

Bruce E. Woodgate and Larry W. Brown

NASA/GSFC Lab for Astronomy \& Solar Physics, Greenbelt, $M D \quad 20771$

\begin{abstract}
The Goddard Fabry-Perot Imager (GFPI) is an optical scanning interferometer and CCD imaging system developed at the Goddard Space Flight Center's Laboratory for Astronomy and Solar Physics for use at various astronomical observatories. Besides being transportable, its most notable characteristic is the relatively low spectral resolution ( $3-30 \AA$ bandpass). The instrument may be used in scanning mode for objects with a large range of velocities but is most often employed to match the observed wavelength of a Doppler shifted emission feature for objects with any velocity.
\end{abstract}

\section{Introduction}

The primary use of the GFPI is as a tuneable narrow band imaging system. The relatively low spectral resolution $(3-30 \AA)$ allows the user to isolate faint emission lines; avoiding nearby, potentially contaminating features and excluding unnecessary corrupting sky and continuum light. In addition, the GFPI allows the user to remove the etalon from the optical path to obtain the pure continuum image with an order separation filter $(55-240 \AA)$ defining the bandpass. Recent publications based on GFPI data include the discovery of a high redshift Ly a source near a damped Ly a absorber (Lowenthal et al. 1991), study of extended line emission associated with quasar radio jets (Shastri et al. 1992), the discovery of interacting galaxies in the Bootes' void (Weistrop et al. 1992), and the discovery of a string of extraordinary line-emitting knots in the Crab Nebula (MacAlpine et al. 1994).

Another optimal use of the GFPI is to image a single object with multiple coarse velocity resolution scans across a large range of wavelengths. Successful examples of such use include the tridimensional models of the Crab supernova remnant (Lawrence et al. 1994 \& these proceedings) or the differing velocity systems in the cluster of galaxies centered on NGC 1275 (Caulet et al. 1992). 


\section{Design}

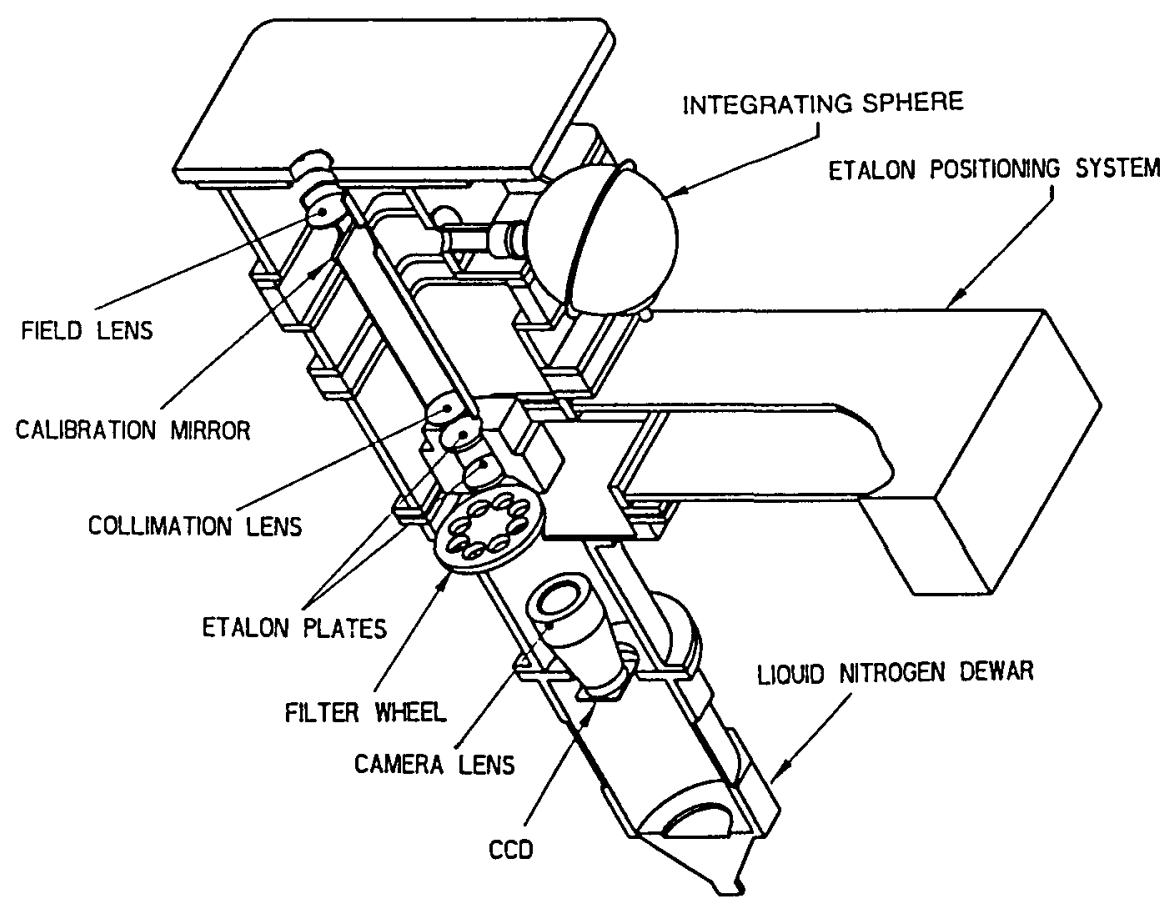

Figure 1. A schematic of the GFPI optical path.

The optical design of the Goddard Fabry-Perot Imager (described in more detail in Brown et al. 1994) is shown in Figure 1. An image at the Cassegrain focus ( $f / 6$ or slower beam) is collimated before passing through the etalons to produce the interference pattern. The beam next passes through a blocking filter with a bandpass smaller than the free spectral range of the desired interference order, restricting the transmittance to a single order of interference. Finally the source is reimaged onto the CCD detector by a $f / 2.8200 \mathrm{~mm}$ camera lens, with a resulting demagnification of 0.65 . A $135 \mathrm{~mm}$ focal length lens is available for greater demagnification (0.44). The resulting field of view and plate scale of the standard detector, a thinned Tektronix $512 \times 512 \mathrm{CCD}\left(27 \mu \mathrm{m} \mathrm{pix}^{-1}\right)$ controlled by a Photometrics camera system, is given in Table 1 for some of the frequently used telescopes. A separate interface protocol allows NOAO CCDs to be used at the KPNO telescopes in place of this detector.

User control of the instrument is achieved through a software interface with the Kinetic Systems camac bus system running on a DEC Alpha computer under the unix-based OSF operating system (a recent upgrade over the previous MicroVAX II computer). The computer also supports the image display, a laser printer, and a read/write optical disk for data storage. Wavelength calibration is performed using emission lines generated by spectral lamps inserted into the 
Table 1. Field of view and pixel scale for GFPI with TK $512^{2}$ CCD.

\begin{tabular}{lcl}
\hline telescope & $\begin{array}{l}\text { (arcminutes) } \\
\text { field of view }\end{array}$ & $\begin{array}{l}\text { (II/pix) } \\
\text { scale }\end{array}$ \\
\hline & & \\
KPNO 4 & & \\
KPNO 2.1 & 2.3 & 0.26 \\
Steward 2.3 & 4.3 & 0.51 \\
MDM 2.4 & 3.5 & 0.41 \\
MDM 1.3 & 4.0 & 0.47 \\
& 7.3 & 0.86 \\
\hline \hline
\end{tabular}

integrating sphere which are directed into the optical path by means of a retractable mirror. The etalon positioning system allows the etalon to be moved in and out of the optical path in a few seconds regardless of telescope position.

The four available etalons (Table 2), manufactured by Queensgate, Ltd., offer complete spectral coverage from 3800 to $10500 \AA$ with variable spectral resolution. These etalons image only the central interferometric ring onto the

Table 2. Characteristics of the four etalons available with the GFPI.

\begin{tabular}{llcc}
\hline Etalon & Range & \multicolumn{2}{c}{ Resolution } \\
& & & \\
Blue & $3800-6000 \AA$ & $3-6 \AA$ & $@ 4600 \AA$ \\
Visible & $4000-7800 \AA$ & $15-30 \AA$ & $@ 6600 \AA$ \\
Red & $5400-7500 \AA$ & $6-12 \AA$ & $@ 6600 \AA$ \\
Far-Red & $6500-10500 \AA$ & $15-30 \AA$ & $@ 6600 \AA$
\end{tabular}

CCD, providing a nearly monochromatic field which varies by a fraction of the spectral FWHM from center to edge. At any given wavelength the spectral resolution and free spectral range are determined by the choice of interference order observed, with narrower bandpasses obtained with higher orders.

The initial wavelength calibration and parallelism adjustment, required due to changes caused by transporation and differing site environment, takes up to three daytime hours after the instrument is installed. For most observing runs there is no need to repeat the calibration, the active servo-stabilization of the 
etalon gap $(\lambda / 200)$ and the temperature control of the GFPI $\left(0.5^{\circ} \mathrm{C}\right)$ provide a spectral stability of better than $1 \AA$.

\section{Availability}

The GFPI has been used on KPNO telescopes since 1990 and continues to be an available choice of instrument for any proposer, with no formal collaboration required (NOAO newsletter \#27, September 1991). A recent run on the European Southern Observatory's $3.6^{\mathrm{m}}$ telescope means the GFPI is now a successfully international instrument. Various collaborations have also brought the instrument to a number of private observatories: the University of Arizona's Steward observatory, the University of Texas' McDonald observatory, and the Michigan-Dartmouth-MIT observatory.

\section{References}

Brown, L.W., Woodgate, B.E., Ziegler, M.M., Kenny, P.J., and Oliversen, R.J. 1994, Review of Scientific Instruments, in press

Caulet, A., Green, R., Woodgate, B., Brown, L., Gull, T., Hintzen, P., Lowenthal, J., Oliversen, R., and Ziegler, M. 1992, ApJ, 388, 301

Lawrence, S.S., MacAlpine, G.M., Uomoto, A., Gelderman, R., Woodgate, B.E., and Brown, L.W. 1994, these proceedings

Lawrence, S.S., MacAlpine, G.M., Uomoto, A., Woodgate, B.E., Brown, L.W., and Oliversen, R.J. 1994, in preparation

Lowenthal, J.D., Hogan, C.J., Green, R.F., Caulet, A., Woodgate, B., Brown, L., and Foltz, C.B. 1991, ApJ, 377, L73

MacAlpine, G.M., Lawrence, S.S., Uomoto, A., Woodgate, B.E., Brown, L.W., Oliversen, R.J., and Lowenthal, J. 1994, ApJ, in press

Shastri, P., Brown, L.W., Wills, D., and Wills, B.J. 1992, in "Physics of Active Galactic Nuclei", Springer-Verlag. p.424

Weistrop, D., Hintzen, P., Kennicutt, R., Liu, C., Lowenthal, J., Cheng, K-P, Oliversen, R., and Woodgate, B. 1992, ApJ, 396, L23 